\title{
Local electronic properties of carbon nanotube heterojunctions
}

\author{
M. S. Ferreira, T. G. Dargam, R. B. Muniz, and A. Latgé* \\ Instituto de Física, Universidade Federal Fluminense, 24210-340, Niterói-RJ, Brazil \\ (Received 5 January 2000; revised manuscript received 9 August 2000)
}

\begin{abstract}
Local electronic properties of metallic-semiconducting carbon nanotube heterostructures are investigated by studying the behavior of the one-electron local density of states (LDOS) along the tubes. We determine how these properties change from the metallic to the semiconducting side of a nanotube junction. We show that Friedel oscillations may not always be evident on the metallic side, and we found clear exponential decay of the LDOS on the semiconducting side. The exponential rates of decay as well as the absence of the oscillations are explained in terms of a simple picture that relates the LDOS to the bulk electronic structure of the constituent parts of the heterostructures.
\end{abstract}

\section{INTRODUCTION}

A large effort in understanding the physical properties of carbon nanotubes ${ }^{1,2}$ (CN's) is now in focus. These tubes are typically longer than $1 \mu \mathrm{m}$, with diameters ranging from 1 to $30 \mathrm{~nm},{ }^{2}$ and are classified as quasi-one-dimensional systems. CN's are presented in a wide variety of geometries showing interesting transport and electronic properties. The most remarkable of those properties is the possibility of having either metallic or semiconducting behavior, depending merely on geometrical aspects of the $\mathrm{CN}$ structure. In fact, experimental results have confirmed such dependence of the electronic structure on the geometry of the tubes. 3,4

The possibility of joining two or more distinct nanotubes enables one to produce tubular heterostructures, offering new perspectives for nanoelectronic technology. Very recently, controlled catalytic growth of junctions between carbon nanotubes and silicon nanowires has been reported. ${ }^{5} \mathrm{~A}$ new type of quantum dot based solely on carbon atoms has been proposed, ${ }^{6}$ motivated by experimental observations of energy quantization in single-walled nanotubes with metallic contacts. ${ }^{7}$ In particular, a metal-semiconductor junction can be made by joining two nanotubes characterized by distinct chiral vectors. One example is the junction of $(n, 0)$ and $(n$ $-1,0)$ zigzag nanotubes, $n$ being an integer multiple of three that defines the chiral vectors. ${ }^{8}$ This type of junction can be made by introducing a single pentagon-heptagon $(\mathrm{PH})$ pair defect along the axial direction of the structure. Structural and electronic properties of zigzag nanotubes, containing $\mathrm{PH}$ pair defects, have been investigated by Charlier et al., ${ }^{9}$ performing tight-binding molecular-dynamics relaxation calculations. They found that junctions whose defects are aligned along the axial direction of the tubes are more stable than those with defects along the circumferential direction. Changes in the chirality of a single-walled nanotube may also occur when $\mathrm{PH}$ defects are aligned neither to the axial nor to the circumferential directions, but in this case, an abrupt bend between two straight sections of the tube is expected. ${ }^{10}$ Alternatively to pure carbon systems, heterostructures based on synthesized $B_{x} C_{y} N_{z}$ nanotubes have also been studied because it is relatively easy to control their electronic properties. ${ }^{11}$
Motivated by the advances in the growth of $\mathrm{CN}$ junctions, and bearing in mind their potential applicability as lowdimensional devices, we have studied local electronic properties of metallic-semiconducting $\mathrm{CN}$ heterostructures. We are particularly interested in investigating how these properties change from the metallic to the semiconducting side of a nanotube junction by studying the behavior of the oneelectron local density of states (LDOS) along the tubes. Far from the junction, the LDOS at the Fermi energy $\left(E_{F}\right)$ must be finite on the metallic side of the tube and vanish on the other. We address the question of how such limits are reached as one moves away from the junction. Friedel-like oscillation and exponential decay of the LDOS at $E_{F}$ are expected on the metallic and semiconducting sides, respectively. Both oscillatory and exponential features can be related to the bulk electronic structure of pure CN's. Friedel oscillations play an important role in long-range interactions in metals and are caused by a drastic change in the density of states at the Fermi energy ${ }^{12}$ at low temperatures. They refer to oscillations of the charge density, but similar oscillations (hereafter referred to as Friedel-like oscillations) occur in the LDOS at $E_{F}$. It is well known that the periods of those oscillations are associated with bulk Fermi-surface wave vectors. Despite the somewhat exotic Fermi surface of metallic CNs, we show that such relation still holds giving rise to Friedel-like oscillations, although they may not always be evident. This is an important point since there is current interest in observing such oscillatory behavior. Although they have been found in short tubes, ${ }^{13}$ the oscillations remain to be observed in long metallic structures. Moreover, we can also determine the rate of decay of the LDOS as we approach the bulk of the semiconducting tube in terms of its electronic structure. In fact, based on the same picture used for determining the periods of Friedel-like oscillations, we relate the exponentially decaying LDOS to evanescent states associated with the so-called complex Fermi surface ${ }^{14,15}$ of the semiconducting tube.

\section{DESCRIPTION OF THE MODEL}

The electronic structure of the nanotube may be obtained from that of two-dimensional graphite. ${ }^{2}$ A single band tightbinding description in terms of $\pi$ orbitals yields a very good 
approximation for the band structure around the Fermi energy. Graphene is semimetallic and its Fermi surface consists of six points at the corners of the two-dimensional Brillouin zone. Since CN's are rolled-up graphene sheets, periodic boundary conditions quantize the components of the electronic wave vectors along the circumferential direction. The axial components, on the other hand, remain continuous for an infinitely long nanotube ${ }^{16}$ and the corresponding electronic states form quasi-one-dimensional energy bands. It should be stressed that such a description of the electronic structure in reciprocal space is useful for studying the properties of infinite pure tubes. ${ }^{8}$ What determines if a $\mathrm{CN}$ is metallic or not is whether the quantized components of the electronic wave vectors along the circumferential direction intersect or not the graphene Fermi surface. ${ }^{2}$ For example, $(n, 0)$ zigzag CN's are metallic only when $n$ is a multiple of three, because only in this case do the quantized $k$ vectors cross the vertices of the hexagonal Brillouin zone of the graphene sheet.

When dealing with finite portions of a $\mathrm{CN}$, or with heterogeneous structures joined by two or more parts, it is convenient to describe the electronic structure in real space. In this case, the single-band tight-binding Hamiltonian of the nanotube can be written in terms of unit cells made of a sequence of spiral bonds between the carbon atoms. In the particular case of achiral CN's, namely armchair and zigzag structures, it is convenient to consider unit cells along the axial direction comprised of two rings associated with the two carbon atoms in the unit cell of the graphene structure. The number of carbon atoms in those rings depends on the $\mathrm{CN}$ diameter. We can treat the Hamiltonian of the system entirely in real space considering a chain of double-ring unit cells. Here we use a real-space renormalization technique to calculate the LDOS at each atom along the heterostructure. We first calculate the surface Green functions for a semiinfinite $\mathrm{CN}$ through the solution of matrix Dyson equations obtained by successive decimations ${ }^{17}$ of unit cells until a fixed point is attained. Having evaluated separately the surface Green functions for a metallic and a semiconducting $\mathrm{CN}$, they are connected by a junction that consists of an axial $\mathrm{PH}$ defect and the necessary number of rings for matching the two semi-infinite parts. It is worth noticing that the distance between the pentagon and heptagon determines the diameter reduction across the junction. The connection of the two sides is theoretically performed using the Dyson equation to include the appropriate sequence of rings of the particular junction joining the two semi-infinite parts. Following this procedure, we can determine the Green functions $G_{l, m}(\omega)$ representing the propagator for electrons with energy $\omega$ between rings $l$ and $m$. We point out that the Green function $G_{j, j}\left(E_{F}\right)$ is a matrix whose size depends on the number of carbon atoms of the ring. The average LDOS at the Fermi level associated with ring $j$ along the joined structure is then obtained by $\rho_{j}\left(E_{F}\right)=-\operatorname{Im} \operatorname{tr} G_{j, j}\left(E_{F}\right) /\left(\pi N_{j}\right)$, where tr stands for the trace over the $N_{j}$ carbon atoms of ring $j$.

\section{NUMERICAL RESULTS}

We calculate the LDOS at $E_{F}=0$ as a function of the ring index for three different heterostructures, all with a single

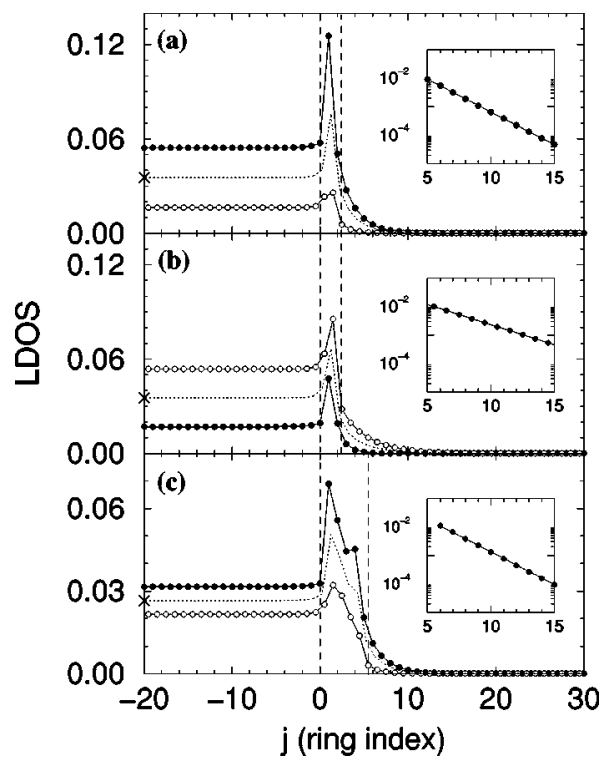

FIG. 1. Local density of states along metal-semiconductor heterojunctions of type (a) $(9,0)-(8,0)$, (b) $(9,0)-(10,0)$, and (c) $(12,0)$ $(8,0)$ as a function of the ring indices and at the Fermi energy $E_{F}$ $=0$. Filled and open circles are associated with rings labeled by integer and half-integer indices, respectively. The crosses indicate the bulk LDOS of the corresponding metallic $\mathrm{CN}$ and dotted curves are guides for the eye showing the unit-cell-averaged LDOS. Dashed lines correspond to the junction limits. The same results for the semiconductor tubes are presented in the inset of the figure but in a logarithmic scale.

junction connecting metallic to semiconducting zigzag CN's. In Figs. 1(a) and 1(b) we show results for metallic $(9,0) \mathrm{CN}$ 's connected to semiconducting $(8,0)$ and $(10,0)$ tubes, respectively. Since the diameter reduction of such structures is the same, both junctions have a common width. Also, in Fig. 1 (c) we present results for the LDOS of a $(12,0) /(8,0)$ heterostructure, which has a wider junction. In all cases the junction region is marked by vertical dashed lines that separate the metallic $(j<0)$ from the semiconducting $(j>W)$ tubes, where $W$ represents the width of the junction. Since the unit cell along the axial direction contains two rings, we have plotted two curves, for integer and half-integer ring indices, depicted by filled and open circles, respectively. It could be argued that the two curves should coincide as one moves away from the junction. In fact, on the semiconducting side they both vanish exponentially. However, on the metallic side the curves tend to distinct limits, which are different from its bulk value. This is a consequence of breaking the axial inversion symmetry of the $\mathrm{CN}$ lattice. For pure semi-infinite CN's, for instance, such symmetry breaking leads to two inequivalent rings even when they are infinitely far from the surface. The bulk LDOS is the average between those of the two inequivalent rings. Since the upper and lower curves in each figure have basically the same behavior, either of them can be chosen to describe how the limiting values of the LDOS are approached. On the metallic side, contrary to expectations, we find no oscillations in the LDOS in all three cases shown in Fig. 1. The oscillations in the LDOS associated with adjacent rings (labeled by integer and half-integer indices, respectively) do not correspond to Friedel-like oscillations. They just reflect the existence of 
two inequivalent rings in the unit cell along the axial direction of a semi-infinite zigzag $\mathrm{CN}$. We will show that the Friedel oscillations in the metallic $\mathrm{CN}$ have a period equal to the unit-cell size (containing two rings), thus being commensurate with the lattice. The LDOS at the Fermi energy on the semiconducting side of the junction is finite near the interface, decaying exponentially as one moves deep into the semiconductor. This is clearly demonstrated for all cases by the straight lines of the insets showing the corresponding LDOS in logarithmic scale. It is noteworthy that the exponential rates of decay depend on the diameter of the semiconducting $\mathrm{CN}$ attached to the junction. The dotted line in Fig. 1 is a guide to the eyes representing the average LDOS over a unit cell. In both sides and sufficiently far from the junction it correctly approaches the corresponding limiting values. Based on a simple model, we show how our results can be explained and related to the electronic structure of the CN's.

\section{ANALYTICAL METHOD AND DISCUSSION}

We are interested in determining the dependence of $\rho_{j}\left(E_{F}\right)$ on the ring index $j$. We start by looking at this dependence for $j$ on the metallic side. By introducing an imaginary cleavage plane separating the metallic side from the rest of the system, we can show that $G_{j, j}$ is given by the following matrix equation:

$$
G_{j, j}=\mathcal{S}_{j, j}+\mathcal{S}_{j,-1} \mathcal{W} \mathcal{S}_{-1, j}
$$

where $\mathcal{S}_{i, j}$ represents the Green-function matrix connecting rings $i$ and $j$ of the semi-infinite metallic $\mathrm{CN}$, and index -1 labels its surface ring. Here, $\mathcal{W}$ involves the surface Green functions of both cleaved parts, namely the metallic side and the rest of the heterojunction. It is worth noticing that $\mathcal{W}$ contains only surface terms and hopping matrices joining the cleaved parts. Therefore, the dependence of $G_{j, j}$ on $j$ is entirely in the $\mathcal{S}$ matrices above. It is possible to show that $\mathcal{S}_{j, j}, \mathcal{S}_{j,-1}$, and $\mathcal{S}_{-1, j}$ can all be expressed in terms of the Green functions $\mathcal{I}$ of the corresponding infinite metallic $\mathrm{CN}$. The $j$ dependence of those $\mathcal{S}$ matrices are then given by the off-diagonal Green function $\mathcal{I}_{-1, j}$, representing the electronic propagator between rings -1 and $j$ for the infinite metallic CN. Since such infinite $\mathrm{CN}$ has axial translation symmetry, $\mathcal{I}_{j, j^{\prime}}$ depends only on the relative positions $\mid j$ $-j^{\prime} \mid$. The actual dependence of $G_{j, j}$ on $j$ is described by the product $\mathcal{I}_{-1, j} \times \mathcal{I}_{j,-1}$. Therefore, to study the position dependence of the LDOS in the heterostructure, one needs to investigate how the off-diagonal propagators $\mathcal{I}_{j, j^{\prime}}$ of infinite pure CN's vary with the ring indices, and, to some extent, this can be done analytically. Although the discussion above is for $j$ on the metallic side, it also applies with minor changes for $j$ on the semiconducting side, the basic difference being that $\mathcal{I}$ is the Green functions of the corresponding infinite semiconducting $\mathrm{CN}$.

In what follows we consider an infinite zigzag nanotube. As mentioned before, in that case it is convenient to describe the electronic structure in reciprocal space. The off-diagonal Green function representing electronic propagation between rings $j$ and $j^{\prime}$ with energy $\omega$ is given by ${ }^{18}$
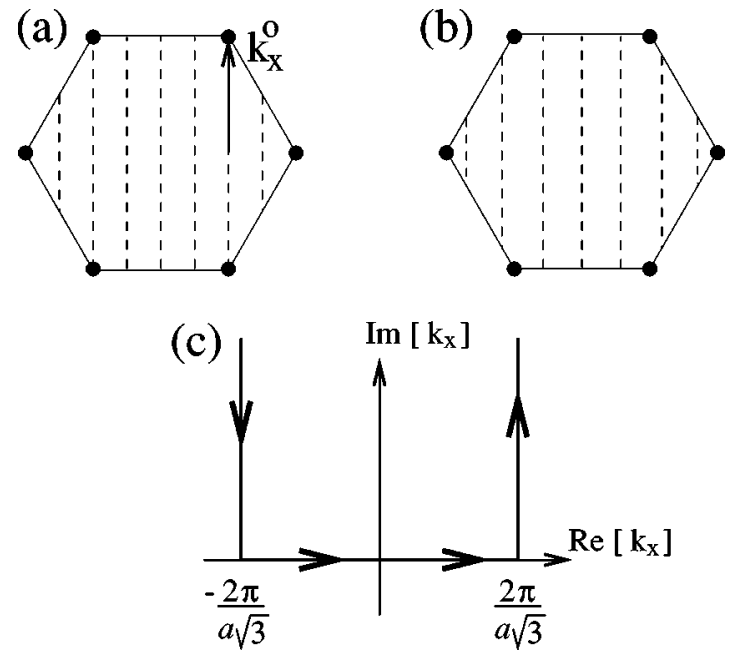

FIG. 2. Two-dimensional Brillouin zones of graphene showing the quantized $k_{y}$ values (dashed lines) for (a) metallic and (b) semiconducting CN's. The real pole $k_{x}^{0}$ corresponding to half the distance between the points of the Fermi surface is depicted in the metallic case. (c) The integration contour is considered for evaluating the integral in Eq. (2), for $j>j^{\prime}$. For $j<j^{\prime}$, the contour is in the lower half-plane.

$$
\mathcal{I}_{j, j^{\prime}}(\omega)=\frac{a \sqrt{3}}{8 \pi} \sum_{k_{y}} \int d k_{x} \frac{2 \omega^{+} e^{i k_{x}\left(j^{\prime}-j\right) a \sqrt{3} / 2}}{\left(\omega^{+}\right)^{2}-\left[E\left(k_{x}, k_{y}\right)\right]^{2}},
$$

where $a$ is the distance between two equivalent sites of the hexagonal lattice $(a=1.42 \sqrt{3} \AA), \omega^{+}=\omega+i \eta, \eta$ is a small imaginary part added to the energy, and $E$ is given by

$$
\begin{aligned}
E\left(k_{x}, k_{y}\right)= & t\left[1+4 \cos \left(\sqrt{3} k_{x} a / 2\right) \cos \left(k_{y} a / 2\right)\right. \\
& \left.+4 \cos ^{2}\left(k_{y} a / 2\right)\right]^{1 / 2},
\end{aligned}
$$

where $t$ is the electronic hopping.

Here $k_{y}$ assumes discrete values, namely $k_{y}$ $=l / N_{y}(2 \pi / a), l=1,2, \ldots, N_{y}$, and $N_{y}$ is determined by the $\mathrm{CN}$ diameter. The $k_{x}$ component, on the other hand, is a continuous variable inside the two-dimensional Brillouin zone. Thus, we end up with a set of one-dimensional bands along $k_{x}$ corresponding to discrete values of $k_{y}$. As previously discussed, the nanotube is metallic if some of the discrete $k_{y}$ lines in reciprocal space intersect the corners of the hexagonal Brillouin zone. This is illustrated in Figs. 2(a) and 2(b), showing discrete $k_{y}$ values represented by vertical dashed lines for a metallic and a semiconducting $\mathrm{CN}$, respectively.

The integral in Eq. (2) can be evaluated for $j<j^{\prime}$ by extending $k_{x}$ to the complex plane and changing the integration contour from a straight line on the real axis to the boundaries of a semi-infinite rectangle in the upper half-plane ${ }^{18}$ whose base lies on the real axis between $-2 \pi / a \sqrt{3}$ and $2 \pi / a \sqrt{3}$, as shown in Fig. 2(c). For the case $j>j^{\prime}$, the extension is to a similar rectangle in the lower half-plane. In either case, the integrand vanishes as $\left|\operatorname{Im} k_{x}\right| \rightarrow \infty$, and because $k_{x}=-(2 \pi / a \sqrt{3})+i y$ and $k_{x}$ $=(2 \pi / a \sqrt{3})+i y$ are equivalent wave vectors, the integrals along the vertical sides of the rectangle cancel each other, reducing the problem to a sum of the residues associated with the poles of the integrand inside the corresponding 
closed contour. The poles are in turn given by the values of $k_{x}$ for which $\left(\omega^{+}\right)^{2}-\left[E\left(k_{x}, k_{y}\right)\right]^{2}=0$. Since we are interested in the LDOS at $E_{F}=0$, the energy $\omega^{+}$is given by $\omega^{+}=0+i \eta$.

The poles are clearly dependent on the band structure since they involve the dispersion relation $E\left(k_{x}, k_{y}\right)$ and are given by

$$
\cos \left(\frac{k_{x}^{0} a \sqrt{3}}{2}\right)=\frac{-1-4 \cos ^{2}\left(\frac{k_{y} a}{2}\right)}{4 \cos \left(\frac{k_{y} a}{2}\right)} .
$$

It is interesting to look at the physical significance of these poles. Each value of $k_{y}$ yields a corresponding $k_{x}$ and since they are obtained from the band structure at $E_{F}$, they are just the coordinates of the $\mathrm{CN}$ Fermi surface along the axial direction [see Fig. 2(a)]. In other words, they indicate the wave vectors $k_{x}^{0}$ with which electrons at the Fermi level propagate along the axial direction. Since the Fermi surface of the metallic $\mathrm{CN}$ is a collection of six isolated points, many discrete values of $k_{y}$ have no real $k_{x}$ components. This happens when $\left|\cos \left(k_{x}^{0} a \sqrt{3} / 2\right)\right|>1$ and $k_{x}^{0}$ assumes imaginary values. The residues associated with the poles are

$$
\operatorname{Res}\left[k_{x}^{0}\right]=\frac{-i \eta e^{i k_{x}^{0}\left(j^{\prime}-j\right) a \sqrt{3} / 2}}{a \sqrt{3} \cos \left(\frac{k_{y} a}{2}\right) \sin \left(k_{x}^{0} a \sqrt{3} / 2\right)} .
$$

The position dependence of the residue is entirely given by the difference $\left(j^{\prime}-j\right)$ which appears in the argument of the exponential. Hence, for real $k_{x}^{0}$, the residue oscillates as a function of $\left(j^{\prime}-j\right)$, whereas it decays exponentially when $k_{x}^{0}$ is imaginary. For a metallic CN, the sequence of discrete $k_{y}$ is such that there is always a real pole $k_{x}^{0}$ that corresponds to half the distance between the points of the Fermi surface, as shown in Fig. 2(a). All the others, for not intersecting the Fermi surface, yield imaginary poles and contribute with evanescent terms. After summing over all $k_{y}$ it is clear that the dominant contribution is the oscillatory term. The reason why no oscillations were found in the metallic side of Fig. 1 becomes clear by recalling that the LDOS depends on the product $\mathcal{I}_{1, j} \times \mathcal{I}_{j, 1}$. Since each Green function contributes with the same oscillation period, the product depends on a wave vector twice as large as the real pole $k_{x}^{0}$, i.e., of the same size as the distance between the points of the Fermi surface. This distance corresponds to the length of the Brillouin zone along the $k_{x}$ direction; therefore, any oscillation with this wave vector cannot be observed for being perfectly commensurate with the lattice. In other words, Friedel-like oscillations are present but are not seen in Fig. 1 because their oscillation period is exactly equal to the unit-cell size containing two rings. The oscillation period in this case is commensurate with the $\mathrm{CN}$ lattice, and is not observable by probing the average LDOS at discrete successive rings. When the probe resolution is sufficiently sharp to identify each ring separately, the LDOS oscillation from one ring to its nearest neighbor may be detected. However, this is not a Friedel-like oscillation, but a phase shift due to the existence of two rings in the unit cell along the axial direction of the
$\mathrm{CN}$. This is a particular case of the so-called aliasing effect, where periods obtained from a discrete sampling of a continuous oscillation differ from the original ones. Such an effect has been addressed before in the study of oscillatory interlayer exchange coupling in magnetic multilayers. ${ }^{19}$

Based on the same picture, we can evaluate the offdiagonal Green functions $\mathcal{I}_{j, j}$, for an infinite semiconducting CN. Each discrete value of $k_{y}$ yields imaginary poles $k_{x}^{0}$, so that we end up with a sum over evanescent states, all exponentially decaying as functions of $\left|j^{\prime}-j\right|$. Clearly, the dominant contribution is the one with the slowest rate of decay. In other words, from all those evanescent states, the one with the smallest imaginary pole $k_{x}^{0}$ determines the rate of decay of $\mathcal{I}_{j, j^{\prime}}$. The rate of decay is given by $2 k_{x}^{0}$ because the LDOS also depends on the product of two similarly decaying functions, as previously discussed. Whereas in the metallic $\mathrm{CN}$ the contributory $k_{y}$ is the one whose quantization line intersects the points of the Fermi surface, in the semiconducting case the dominant evanescent state will be the one closest to that line. In fact, there is perfect agreement between the slopes of the LDOS in logarithmic scale in the insets of Fig. 1 and the values we have calculated. For the particular case of a $(8,0)$ nanotube the exponentially decaying LDOS behaves as $e^{-\lambda j}$ as a function of $j$, where $\lambda$ $=0.53$ is found both analytically and numerically. The agreement is also perfect for the semiconducting $(10,0) \mathrm{CN}$, where the rate of decay is found to be $\lambda=0.32$. This indicates unambiguously that the asymptotic rate of decay is completely determined by the bulk electronic structure of the particular semiconducting tube and depends neither on the details of the junction nor on its metallic counterpart.

In metallic systems, a few portions of their bulk Fermi surfaces determines the periods of Friedel-like oscillations. We have extended this picture to relate, not only the periods, but also the rate of decay of the LDOS of a heterojunction to the electronic structure of a semiconducting CN. Instead of being associated with extended electronic states of the Fermi surface, the dominant evanescent states giving the exponentially decaying LDOS are related to the so-called complex Fermi surface, ${ }^{14,15}$ consisting of imaginary wave vectors.

\section{DOPED NANOTUBES $\left(E_{F} \neq 0\right)$}

Whereas isolated nanotubes have their Fermi energies predicted at $E_{F}=0$, a charge-transfer-induced shift in $E_{F}$ may occur when the tube is deposited onto a substrate. Moreover, the ability to vary the Fermi energy in a $\mathrm{CN}$ has been previously discussed. ${ }^{7,20-22}$ To test the validity of our method, we have slightly changed the Fermi energy of the $(12,0) /(8,0)$ junction. As a result, the Fermi surface of metallic CN's becomes small pockets centered at the six corners of the two-dimensional Brillouin zone. In this case, we no longer have periods perfectly commensurate with the lattice, and the oscillations are clearly seen. We consider two distinct values for the Fermi energy, namely $E_{F}=0.05$ and $E_{F}$ $=0.1$, in units of the hopping integral. Figure 3 shows the LDOS for those different values of $E_{F}$ as a function of the ring index. Figures 3(a) and 3(b) correspond to the metallic parts whereas 3(c) and 3(d) show the LDOS in logarithmic scale along the semiconducting sides. Both values of $E_{F}$ show clear oscillations on the metallic side whose periods 

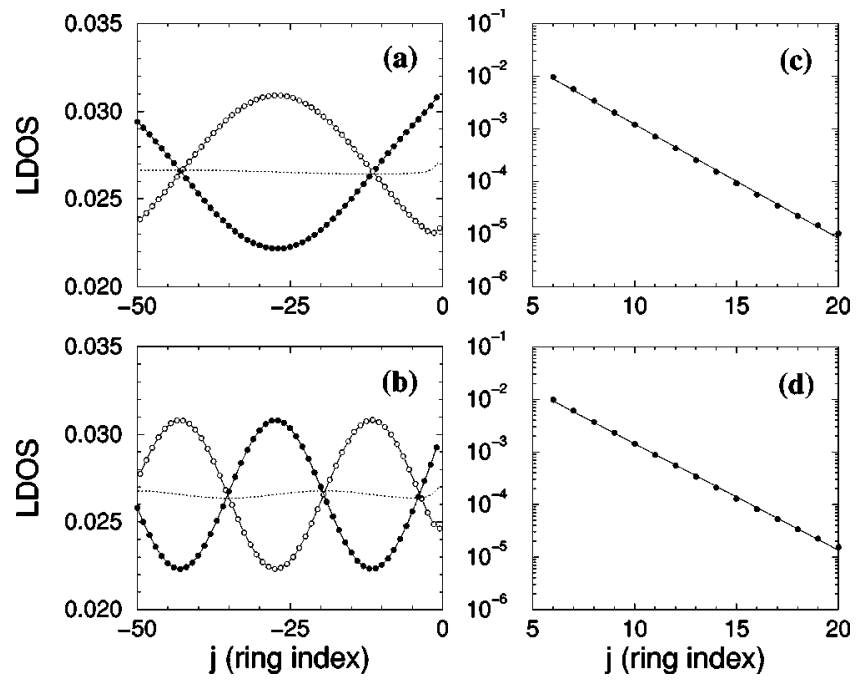

FIG. 3. Local density of states along a $(12,0)-(8,0)$ heterojunction for the [(a) and (b)] metal side and [(c) and (d)] semiconductor tube as a function of the ring index and for two values of the Fermi energy: $E_{F}=0.05$ in (a) and (c), and $E_{F}=0.1$ in (b) and (d); energies are given in units of the hopping integral. The dotted curves in (a) and (b) are guides for the eye showing the unit-cell-averaged LDOS.

coincide exactly with the calculated values. For slight changes around $E_{F}=0$, the oscillation periods decrease with $\left|E_{F}\right|$, reflecting the enlargement of the Fermi-surface pockets around the corners of the two-dimensional Brillouin zone. We recall that charge-density oscillations in one dimension decay away from the interface but not the corresponding oscillations in the LDOS, whose amplitudes remain constant, as can be clearly seen in Figs. 3(a) and 3(b). It is worth stressing that the exponential rate of decay is also affected but the calculated values from the corresponding poles remain in perfect agreement with the numerical observations. On the metallic side, we point out that even when the periods are not commensurate with the lattice, intracell phase shifts may hide the Friedel-like oscillations. When the LDOS is averaged over the unit cell, their amplitude is strongly reduced (due to the phase shift) as shown in Figs. 3(a) and 3(b). Therefore, measurements probing such an average may experience difficulties in detecting the Friedel oscillations.
Experimental techniques capable of distinguishing intracell rings (such as atomic resolved microscopy) may be more suitable to making such oscillations evident, owing to the fact that they only become apparent when the LDOS is plotted as a function of the number of unit cells.

\section{CONCLUSIONS}

We have investigated the electronic LDOS at the Fermi level across heterostructures composed of two attached metallic and semiconducting semi-infinite CN's. No Friedel-like oscillations were observed on the metallic side, whereas clear exponential decays are seen on the semiconducting one. Based on a simple picture that describes the heterostructure in terms of infinite metallic or semiconducting CN's, the asymptotic behavior of the LDOS was related to the bulk electronic structure of its constituent parts, being independent of the intervening junction. More precisely, the oscillatory behavior of the LDOS was associated with particular wave vectors of the Fermi surface of metallic CN's. Also, by extending the same picture to account for evanescent states, the exponential rate of decay on the semiconducting side was associated with imaginary wave vectors of the complex Fermi surface. The perfect agreement of the analytically evaluated periods and decay rates with the numerical results confirms the validity of the present model calculation. Furthermore, the absence of oscillations on the metallic side was shown to result from periods commensurate with the distance between the carbon rings. The oscillations do exist but are hidden by periods that coincide with the lattice spacing. In fact, as the Fermi energy is slightly changed, noncommensurate periods arise and the oscillations of the LDOS become apparent. Measurements of the Friedel-like oscillation periods on doped carbon nantubes provide information about the Fermi-level shift in these structures. Since transport phenomena across heterogeneous nanotube structures are believed to be promising for technological applications, the understanding of their local electronic properties is essential for further development in this field.

\section{ACKNOWLEDGMENTS}

The authors would like to thank CNPq and FAPERJ of Brazil for partial financial support.
*Electronic address: latge@if.uff.br

${ }^{1}$ S. Ijima, Nature (London) 354, 56 (1991).

${ }^{2}$ M. S. Dresselhaus, G. Dresselhaus, and P. C. Eklund, Science of Fullerenes and Carbon Nanotubes (Academic Press, New York, NY, 1996); R. Saito, G. Dresselhaus, and M. S. Dresselhaus, Physical Properties of Carbon Nanotubes (Imperial College Press, London, 1998).

${ }^{3}$ J.W.G. Wildoer, L.C. Venema, A.G. Rinzler, R.E. Smalley, and C. Dekker, Nature (London) 391, 59 (1998).

${ }^{4}$ T.W. Odom, J.-L. Huang, P. Kim, and C.M. Lieber, Nature (London) 391, 62 (1998).

${ }^{5}$ J. Hu, M. Ouyang, P. Yang, and C.M. Lieber, Nature (London) 399, 48 (1999).

${ }^{6}$ L. Chico, M.P. López Sancho, and M.C. Munoz, Phys. Rev. Lett. 81, 1278 (1998).
${ }^{7}$ S.J. Tans, M.H. Devoret, H. Dai, A. Thess, R.E. Smalley, L.J. Geerligs, and C. Dekker, Nature (London) 386, 474 (1997); D.H. Cobden, M. Bockrath, N. Chopra, A. Zettl, P. McEuen, A. Rinzler, A. Thess, and R.E. Smalley, Physica B 251, 132 (1998).

${ }^{8}$ M.S. Dresselhaus, G. Dresselhaus, and R. Saito, Phys. Rev. B 45, 6234 (1992).

${ }^{9}$ J.-C. Charlier, T.W. Ebbesen, and Ph. Lambin, Phys. Rev. B 53, 11108 (1996).

${ }^{10}$ L. Chico, V.H. Crespi, L.X. Benedict, S.G. Louie, and Marvin L. Cohen, Phys. Rev. Lett. 76, 971 (1996).

${ }^{11}$ X. Blase, J.-C. Charlier, A. De Vita, and R. Car, Appl. Phys. Lett. 70, 197 (1996).

${ }^{12} \mathrm{Ph}$. Hofmann, B.G. Briner, M. Doering, H.-P. Rust, E.W. Plummer, and A.M. Bradshaw, Phys. Rev. Lett. 79, 265 
(1997).

${ }^{13}$ L.C. Venema, J.W.G. Wildoer, J.W. Janssen, S.J. Tans, H.L.J.T.Tuinstra, L.P. Kouwenhoven, and G. Dekker, Science 283, 52 (1999).

${ }^{14}$ P. Bruno and C. Chappert, Phys. Rev. Lett. 67, 1602 (1991).

${ }^{15}$ M.S. Ferreira, J. d'Albuquerque e Castro, R.B. Muniz, and L.C. Lopez, Phys. Rev. B 58, 8198 (1998); V. Heine, Proc. Phys. Soc. London 81, 300 (1963).

${ }^{16}$ Supriyo Datta, Electronic Transport in Mesocopic Systems (Cambridge University Press, Cambridge, 1997).

${ }^{17}$ D.C. Marcucci, A. Latgé, E.V. Anda, M. Matos, and
J.C.Fernandes, Phys. Rev. B 56, 3672 (1997).

${ }^{18}$ M.S. Ferreira, J. d'Albuquerque e Castro, D.M. Edwards, and J. Mathon, J. Phys.: Condens. Matter 8, 11259 (1996).

${ }^{19}$ R. Coehoorn, Phys. Rev. B 44, 9331 (1991).

${ }^{20}$ M.P. Anantram and T.R. Govindan, Phys. Rev. B 58, 4882 (1998).

${ }^{21}$ M. Bockrath, D.H. Cobden, P.L. McEuen, N.G. Chopra, A. Zettl, A. Thess, and R.E. Smalley, Science 275, 1922 (1997).

${ }^{22}$ A. Bezryadin, A.R.M. Verschreren, S.J. Tans, and C. Dekker, Phys. Rev. Lett. 80, 4036 (1998). 